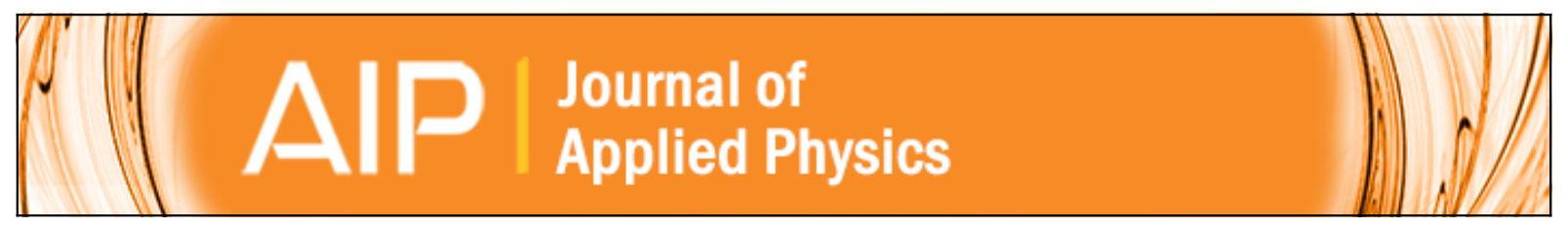

The interaction between a monolayer of single-molecule magnets and a metal surface

Salvador Barraza-Lopez, Michael C. Avery, and Kyungwha Park

Citation: Journal of Applied Physics 103, 07 B907 (2008); doi: 10.1063/1.2830014

View online: http://dx.doi.org/10.1063/1.2830014

View Table of Contents: http://scitation.aip.org/content/aip/journal/jap/103/7?ver=pdfcov

Published by the AIP Publishing

Articles you may be interested in

Charge transfer and formation of reduced $\mathrm{Ce} 3+$ upon adsorption of metal atoms at the ceria (110) surface

J. Chem. Phys. 136, 134703 (2012); 10.1063/1.3697485

Gate-induced switching in single-molecule magnet MnllICull

J. Appl. Phys. 110, 023702 (2011); 10.1063/1.3610448

Spin-filtering effect in the transport through a single-molecule magnet $\mathrm{Mn} 12$ bridged between metallic electrodes J. Appl. Phys. 105, 07E309 (2009); 10.1063/1.3072789

Impacts of metal electrode and molecule orientation on the conductance of a single molecule Appl. Phys. Lett. 85, 5992 (2004); 10.1063/1.1836872

Electronic properties of metal-molecule-metal systems at zero bias: A periodic density functional study J. Chem. Phys. 119, 6729 (2003); 10.1063/1.1602057

MIT LINCOLN LABORATORY CAREERS

Discover the satisfaction of innovation and service to the nation
- Space Control

- Air \& Missile Defense

- Communications Systems \& Cyber Security

- Intelligence, Surveillance and

Reconnaissance Systems

\section{LINCOLN LABORATORY}

MassachusetTS Institute OF TeCHNOLOGY

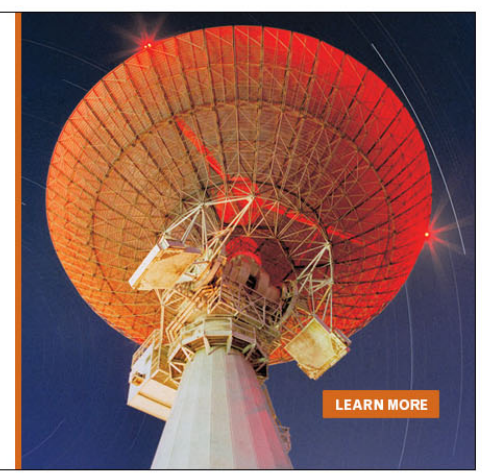




\title{
The interaction between a monolayer of single-molecule magnets and a metal surface
}

\author{
Salvador Barraza-Lopez, ${ }^{\text {a) }}$ Michael C. Avery, and Kyungwha Park ${ }^{\text {b) }}$ \\ Department of Physics, Virginia Polytechnic Institute and State University, Blacksburg, Virginia 24061, USA
}

(Presented on 9 November 2007; received 12 September 2007; accepted 8 October 2007; published online 4 February 2008)

\begin{abstract}
We calculate within density functional theory (DFT) and the LSDA $+U$ formalism the electronic properties of a nanostructure in which single-molecule magnets $\mathrm{Mn}_{12}$ are adsorbed via thiol groups onto the $\mathrm{Au}(111)$ surface. Our DFT calculation shows 1.23 electrons being transferred from the surface to the $\mathrm{Mn}_{12}$ molecule, dominated by the tail on the electronic charge distribution from the gold slab. LSDA $+U$ calculations reveal that the on-site Coulomb repulsion $U$ does not alter the direction of the electronic charge transfer obtained from DFT, because the gold Fermi level still lies above the lowest unoccupied molecular orbital (LUMO). The $U$ term opens up the energy gap between the highest occupied molecular orbital (HOMO) and the LUMO for an isolated standard $\mathrm{Mn}_{12}$ but it minimally affects the gap for a sulfur-terminated $\mathrm{Mn}_{12}$. (C) 2008 American Institute of Physics. [DOI: 10.1063/1.2830014]
\end{abstract}

The recent interest in single-molecule magnets (SMMs) deposited on various surfaces ${ }^{1-5}$ or bridged between electrodes arises from their potential use in magnetoelectronic devices and materials for quantum computing. ${ }^{6-8}$ Among thousands of synthesized SMMs, $\left[\mathrm{Mn}_{12} \mathrm{O}_{12}\left(\mathrm{CH}_{3} \mathrm{COO}\right)_{16}\right.$ $\left.\left(\mathrm{H}_{2} \mathrm{O}\right)_{4}\right]$ (referred to as $\mathrm{Mn}_{12}$ ) (Ref. 9) was extensively studied in bulk and in molecular structures deposited onto a surface or bridged between electrodes, either through attractive van der Waals forces or via ligand exchange with the $\mathrm{Mn}_{12}$ molecules, due to its large magnetic anisotropy barrier of $65 \mathrm{~K}^{10}$ Experimental studies on monolayers of $\mathrm{Mn}_{12}$ molecules on a surface showed that the Mn $3 d$ partial density of states in valence bands remains the same as that for bulk $\mathrm{Mn}_{12}$, but that their magnetic properties change upon adsorption. ${ }^{3-5,11}$ Despite active experimental efforts in understanding the properties of SMMs adsorbed on surfaces, little is known about (i) the orientation of SMMs on surfaces and (ii) the characteristics of the interface between the molecules and surface. The lack of this crucial information demands to examine the electronic and magnetic properties of SMMs interacting with a surface, using atomic-scale simulations.

Motivated by this need, we performed atomic-scale simulations of a monolayer of SMMs $\mathrm{Mn}_{12}$ on a $\mathrm{Au}(111)$ surface, using spin-polarized density-functional theory (DFT). ${ }^{12}$ We used the Perdew-Burke-Ernzerhof (PBE) generalized-gradient approximation ${ }^{13}$ (GGA) and projectoraugmented-wave (PAW) pseudopotentials ${ }^{14}$ within the Vienna $a b$ initio simulation package (VASP). ${ }^{15,16}$ In our DFT calculation, $\mathrm{Mn}_{12}$ molecules [Fig. 1(a)] were oriented such that the magnetic easy axis is normal to the surface, and terminating ligands of the $\mathrm{Mn}_{12}$ molecule were exchanged with thiol groups $(-\mathrm{SH})$, where the $\mathrm{H}$ atoms were lost to form a strong, direct bonding to a gold surface. These sulfurterminated $\mathrm{Mn}_{12}$ molecules were attached to a 6 monolayer

\footnotetext{
${ }^{a)}$ Electronic mail: salva@vt.edu

b)Electronic mail: kyungwha@vt.edu
}

(ML) $\mathrm{Au}(111)$ slab, as shown in Fig. 2(a). The geometry shown in Fig. 2(a) is called the whole structure and the sulfur-terminated $\mathrm{Mn}_{12}$ molecule is called geometry 2 [Fig. $1(b)]$.

Conventional DFT calculations include spurious selfinteractions among electrons, which result in delocalized electrons. $d$ electrons in transition metal compounds are strongly localized due to strong correlation, and thus are highly affected by self-interaction. A practical way to include the self-interaction correction in DFT is to introduce an effective on-site Coulomb repulsion term $U$ within the $\mathrm{LSDA}+U$ formalism $^{17,18}$ (LSDA stands for local spin density approximation). The $U$ term typically increases the electronic gap when compared to DFT results. Charge transfer and electronic transport properties are partly determined by the relative alignment of the lowest unoccupied molecular

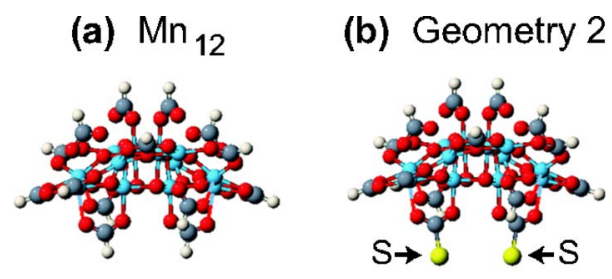

(c) Ground-state magnetic moment

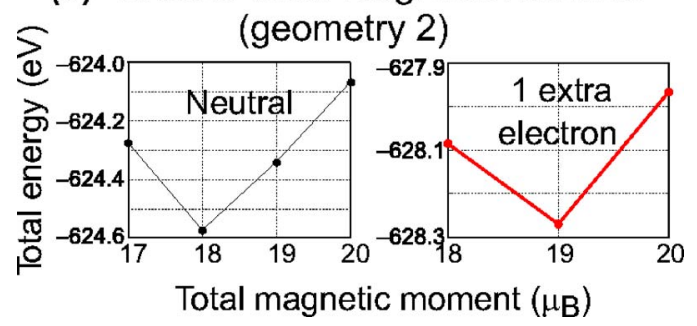

FIG. 1. (Color online) Side views of (a) $\left[\mathrm{Mn}_{12} \mathrm{O}_{16}(\mathrm{HCOO})_{16}\right]\left(\mathrm{Mn}_{1} 2\right)$ molecule and (b) $\left[\mathrm{Mn}_{12} \mathrm{O}_{12}(\mathrm{HCOO})_{14}(\mathrm{SCOO})_{2}\right]$ molecule (geometry 2). (c) Total energy calculated using DFT vs total magnetic moment for neutral and charged molecules with geometry 2 . 


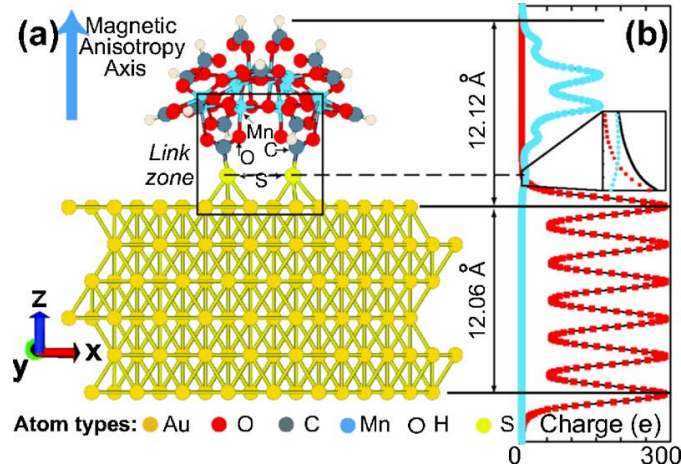

FIG. 2. (Color online) (a) Whole structure composed of a $6 \mathrm{ML}$ gold slab onto which geometry 2 is deposited. (b) Charge profile in the vertical direction: Black solid curve (whole structure), blue dots (isolated geometry 2), and red squares (gold slab). The inset in (b) highlights the penetration of the electron cloud of the gold slab into geometry 2 .

orbital (LUMO) and the highest occupied molecular orbital (HOMO) with the Fermi level of the metal surface. In this work we focus on the effect of the $U$ term on the electronic structure of the whole structure, and compare our result with our previous DFT calculation ${ }^{12}$ and experimental data. ${ }^{5,19,20}$

To facilitate the comparison we briefly discuss our DFT results using GGA and then present $\mathrm{LSDA}+U$ results. For the gold slab, within GGA, the total energy converged with the energy cutoff of $260 \mathrm{eV}$ for plane waves [Fig. 3(a)], and the equilibrium lattice constant $a_{0}=4.175 \AA$ was found [Fig. $3(b)]$. The gold slab was optimized along the vertical direction with the in-plane separation set to $a_{0} / \sqrt{2}$, until forces were less than $0.01 \mathrm{eV} / \AA$. The total energy of the gold slab per monolayer is shown as a function of the number of monolayers in Fig. 3(c). 6 ML have the minimal height to provide the proper electrostatic screening of the molecules. Our DFT calculation showed that the total magnetic moment for a $\mathrm{Mn}_{12}$ molecule is $20 \mu_{B}$, while that for a neutral molecule with geometry 2 is $18 \mu_{B}$, as shown in Fig. 1(c). For geometry $2\left(\mathrm{Mn}_{12}\right)$ the HOMO and LUMO come from $p$ orbitals of the $\mathrm{S}$ atoms ( $d$ orbitals of the $\mathrm{Mn}$ atoms). We calculated the single-electron charging energy for geometry 2 from the energy difference between a neutral molecule and a charged molecule. When one extra electron is added to geometry 2 , the total magnetic moment is modified to $19 \mu_{B}$. Considering this, we found a charging energy of $3.7 \mathrm{eV}$ $(3.9 \mathrm{eV})$ within GGA (LSDA), which is close to $U=4 \mathrm{eV}$ reported in Ref. 20. Geometry 2 was attached to the gold slab and we calculated the electronic charge transfer between the molecule and the surface in the $z$ direction (integrated over the $x-y$ plane), as shown in Fig. 2(b). The tail of the gold slab contributes 1.23 electrons to the molecule. The total mag- netic moment is modified to $20 \mu_{B}$ upon adsorption on the surface.

Now we present our LSDA $+U$ calculations on bulk $\mathrm{Au}$, an isolated $\mathrm{Mn}_{12}$ molecule, and a molecule with geometry 2 . For LSDA calculations we use the exchange correlation as parametrized from Ceperley-Alder data ${ }^{21}$ and PAW pseudopotentials in VASP. We explore suitable effective screened Coulomb $U$ and exchange $J$ parameters from the LSDA $+U$ formalism ${ }^{17,18}$ for $d$-electron atoms. The choice of parameters is justified by experimental data, such as the bulk lattice constant and the HOMO-LUMO gap, as well as our DFT calculation on the charging energy for geometry 2. For bulk gold, only $5 d$ orbitals are affected by the $U$ parameter. The lattice constant obtained from LSDA is $4.063 \AA$, which is closer to the extrapolated $(T \rightarrow 0)$ experimental value, ${ }^{22}$ $4.059 \AA$, than the GGA value, $4.175 \AA$. Using the LSDA $+U$ formalism introduced in Ref. 17, we obtain the lattice constant and the Fermi energy as a function of $U$ (Table I). We find that $U=0.6 \mathrm{eV}$ brings the lattice constant to the extrapolated experimental value. As long as $(U-J)=0.6$, the screened exchange $J$ term does not significantly affect the electronic structure, and thus we use $U=0.6 \mathrm{eV}$ and $J$ $=0 \mathrm{eV}$ for bulk Au, with the LSDA $+U$ method as described in Ref. 17. With the relative small $U=0.6 \mathrm{eV}$ the Fermi energy decreases by only $0.1 \mathrm{eV}$ compared to that with $U=0$.

For a $\mathrm{Mn}_{12}$ molecule and geometry 2 we calculate their electronic structures with $U=4.0$ and $6.0 \mathrm{eV}(J=0.0 \mathrm{eV}$ throughout). The LSDA $+U$ method of Ref. 18 was employed. Photoemission spectra and other LSDA $+U$ calculation suggested to use $U=4.0 \mathrm{eV}$ for $\mathrm{Mn}_{12}$, and our GGA (LSDA) calculation indicated 3.7 (3.9) eV for geometry 2. As shown in Table II, for $\mathrm{Mn}_{12}$ both majority and minority HOMOs are greatly lowered with $U$ to provide a large HOMO-LUMO gap of the order of $1 \mathrm{eV}$, while the majority and minority LUMOs do not change much with $U$. With $U$ $=4$ and $6 \mathrm{eV}$, the total magnetic moment is found to be $20 \mu_{B}$ in the ground state. For geometry 2 the majority and minority HOMO and LUMO are all affected by the $U$ term, but the HOMO-LUMO gaps for majority and minority spins do not change significantly. At $U=4 \mathrm{eV}$, the majority HOMOLUMO gap becomes $0.28 \mathrm{eV}$, which is $0.13 \mathrm{eV}$ larger than without $U$ but still much smaller than the gap found for $\mathrm{Mn}_{12}$. This is due to the nature of the HOMO and LUMO in geometry 2, dominated by the sulfur $p$ orbitals. The overall shift of the orbitals is caused by the effect of $U$ on the Mn $d$ orbitals. Within the LSDA $+U$ formalism, the gold Fermi level is $-4.81 \mathrm{eV}$ with $U_{\mathrm{Au}}=0.6 \mathrm{eV}$, so the gold Fermi level lies well above both majority LUMO and minority LUMO. This indicates that the electronic charge is transferred from
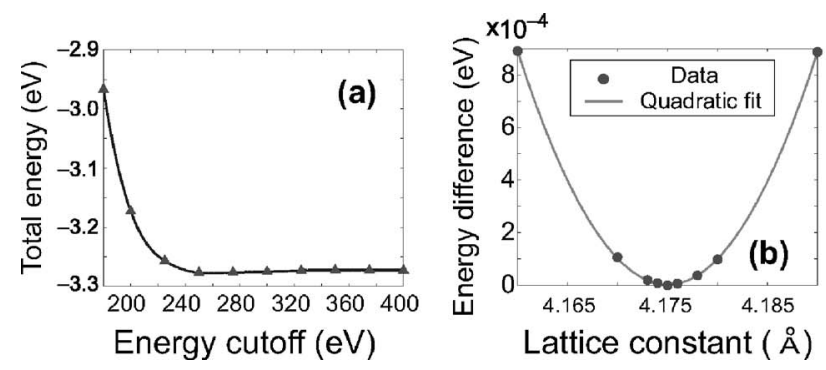

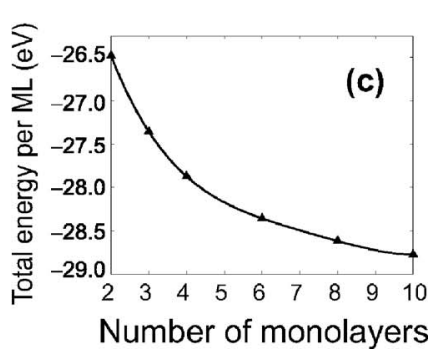

FIG. 3. Determination of the optimum (a) energy cutoff and (b) lattice constant for bulk Au. (c) Convergence of the total energy per monolayer vs the number of monolayers employed. In (c) the height of the unit cell is fixed to $34 \AA$. 
TABLE I. Dependence of the lattice constant and the Fermi energy on the $U$ term $(J=0)$ for bulk Au.

\begin{tabular}{lccccc}
\hline \hline \multicolumn{1}{c}{$U_{\mathrm{Au}}(\mathrm{eV})$} & -0.5 & $0.0(\mathrm{LSDA})$ & 0.5 & 0.6 & 1.0 \\
\hline$a_{0}(\AA)$ & 4.067 & 4.063 & 4.060 & 4.059 & 4.056 \\
$E_{\mathrm{Fermi}}(\mathrm{eV})$ & -4.65 & -4.72 & -4.80 & -4.81 & -4.87 \\
\hline \hline
\end{tabular}

TABLE II. Majority and minority HOMO and LUMO (in eV) from LSDA $+U$ and PBE-GGA calculations.

\begin{tabular}{ccccccc}
\hline \hline \multirow{2}{*}{$\begin{array}{c}\text { Electronic } \\
\text { level }\end{array}$} & \multicolumn{2}{c}{$U=4, J=0$} & \multicolumn{2}{c}{$U=6, J=0$} & \multicolumn{2}{c}{ PBE-GGA $^{\text {a }}$} \\
\cline { 2 - 7 } & Majority & Minority & Majority & Minority & Majority & Minority \\
\hline $\mathrm{Mn}_{12}$ HOMO & -5.80 & -7.04 & -6.13 & -7.04 & -5.08 & -6.45 \\
Mn $_{12}$ LUMO & -4.72 & -4.57 & -4.78 & -4.77 & -4.84 & -4.50 \\
Geo. 2 HOMO & -6.19 & -6.52 & -6.37 & -6.66 & -5.37 & -6.07 \\
Geo. 2 LUMO & -5.91 & -5.29 & -6.05 & -5.51 & -5.22 & -4.84 \\
\hline \hline
\end{tabular}

${ }^{\mathrm{a}}$ Reference 12 .

the surface to the molecule. However, since now the gold Fermi level is also below minority LUMO, the spin polarization in the charge transferred is not maintained. This is in contrast to the PBE-GGA result where the gold Fermi level $(-4.95 \mathrm{eV})$ was well below the majority LUMO but above the minority LUMO, so only electrons with majority spin were transferred. ${ }^{12}$

In conclusion, we have studied, by means of relative level alignments, the interaction between a monolayer of single-molecule magnets $\mathrm{Mn}_{12}$ and a gold surface within the LSDA $+U$ formalism, and compared with our previous DFT calculations. The electronic structure and magnetic properties of the whole structure using the LSDA $+U$ formalism are currently being investigated.

M.C.A. and K.P. were supported by the Jeffress Memorial Trust. Computational support was provided by the SGI Altix Linux Supercluster at the National Center for Supercomputing Applications under Contract No. DMR060009N, and Virginia Tech Inferno 2 and Cauldron systems.

${ }^{1}$ J. S. Steckel, N. S. Persky, C. R. Martinez, C. L. Barnes, E. A. Fry, J. Kulkarni, J. D. Burgress, R. B. Pacheco, and S. L. Stoll, Nano Lett. 4, 399 (2004).

${ }^{2}$ L. Zobbi, M. Mannini, M. Pachioni, G. Chastanet, D. Bonacchi, C. Zanardi, R. Biagi, U. del Pennino, D. Gateschi, A. Cornia, and R. Sessoli, Chem. Commun. (Cambridge) 12, 1640 (2005).

${ }^{3}$ A. Naitabdi, J.-P. Bucher, P. Gerbier, P. Rabu, and M. Drillon, Adv. Mater. (Weinheim, Ger.) 17, 1612 (2005).

${ }^{4}$ S. Voss, M. Fonin, U. Rudiger, M. Burgert, and U. Groth, Appl. Phys.
Lett. 90, 133104 (2007).

${ }^{5}$ S. Voss, M. Fonin, U. Rudiger, M. Burgert, U. Groth, and Y. S. Dedkov, Phys. Rev. B 75, 045102 (2007).

${ }^{6}$ C. Joachim, J. K. Gimzewski, and A. Aviram, Nature (London) 98, 541 (2000).

${ }^{7}$ C. Timm and F. Elste, Phys. Rev. B 73, 235304 (2006).

${ }^{8}$ M. N. Leuenberger and D. Loss, Nature (London) 410, 789 (2001).

${ }^{9}$ T. Lis, Acta Crystallogr., Sect. B: Struct. Crystallogr. Cryst. Chem. 36, 2042 (1980).

${ }^{10}$ A. L. Barra, D. Gatteschi, and R. Sessoli, Phys. Rev. B 56, 8192 (1997).

${ }^{11}$ Z. Salman, K. H. Chow, R. I. Miller, A. Morello, T. J. Parolin, M. D. Hossain, T. A. Keeler, C. D. P. Levy, W. A. MacFarlane, G. D. Morris, H. Saadaoui, D. Wang, R. Sessoli, G. G. Condorelli, and R. F. Kiefl, Nano Lett. 7, 1551 (2007).

${ }^{12}$ S. Barraza-Lopez, M. C. Avery, and K. Park, Phys. Rev. B 76, 224413 (2007).

${ }^{13}$ J. P. Perdew, K. Burke, and M. Ernzerhof, Phys. Rev. Lett. 77, 3865 (1996).

${ }^{14}$ P. E. Blöchl, Phys. Rev. B 50, 17953 (1994).

${ }^{15}$ G. Kresse and J. Furthmüller, Phys. Rev. B 54, 11169 (1996).

${ }^{16}$ G. Kresse and J. Furthmüller, Phys. Rev. B 59, 1758 (1999).

${ }^{17}$ V. I. Anisimov, F. Aryastiawan, and A. I. Lichtenstein, J. Phys.: Condens. Matter 9, 767 (1997).

${ }^{18}$ S. L. Dudarev, G. A. Botton, S. Y. Savrasov, C. J. Humphreys, and A. P. Sutton, Phys. Rev. B 57, 1505 (1998).

${ }^{19}$ U. del Pennino, V. D. Renzi, R. Biaggi, V. Corraldini, L. Zobbi, A. Cornia, D. Gatteschi, F. Bondino, E. Magnano, M. Zangrando, M. Zacchigna, A. Lichtenstein, and D. W. Boukhvalov, Surf. Sci. 600, 4185 (2006).

${ }^{20}$ D. W. Boukhvalov, M. Al-Saqer, E. Z. Kurmaev, A. Moewes, V. R. Galakhov, L. D. Finkelstein, S. Chiuzbăian, M. Neumann, V. V. Dobrovitski, M. I. Katsnelson, A. I. Lichtenstein, B. N. Harmon, K. Endo, J. M. North, and N. S. Dalal, Phys. Rev. B 75, 014419 (2007).

${ }^{21}$ D. M. Ceperley and B. J. Alder, Phys. Rev. Lett. 45, 566 (1980).

${ }^{22}$ A. Khein, D. J. Singh, and C. J. Umrigar, Phys. Rev. B 51, 4105 (1995). 